\title{
Clinicoradiological Features of Recurrent Meningioma With High Grade Transformation
}

\author{
EUNG KOO YEON ${ }^{1}$, JI-YOUN SUNG $^{2}$, SUNG-IM DO $^{3,4}$, BONG JIN PARK $^{5}$, EUI JONG KIM ${ }^{1}$ and KIYONG NA ${ }^{2}$ \\ ${ }^{1}$ Department of Radiology, Kyung Hee University Hospital, \\ Kyung Hee University College of Medicine, Seoul, Republic of Korea; \\ ${ }^{2}$ Department of Pathology, Kyung Hee University Hospital, \\ Kyung Hee University College of Medicine, Seoul, Republic of Korea; \\ ${ }^{3}$ Department of Pathology, Kangbuk Samsung Hospital, \\ Sungkyunkwan University College of Medicine, Seoul, Republic of Korea; \\ ${ }^{4}$ Department of Pathology, City of Hope Comprehensive Cancer Center, Duarte, CA, U.S.A.; \\ ${ }^{5}$ Department of Neurosurgery, Kyung Hee University Hospital, \\ Kyung Hee University College of Medicine, Seoul, Republic of Korea
}

\begin{abstract}
Background/Aim: A minority of grade I meningiomas $(M G 1 s)$ recur after surgical resection and their progression is associated with high grade transformation (HGT). This study aimed to characterize the clinicoradiological features of recurrent MGs (RMG) with HGT. Patients and Methods: We identified 17 patients diagnosed with MG1 who then underwent surgery for RMG. Patients were categorized into HGT group vs. non-HGT (nHGT) group based on RMG histological grade and clinicoradiological features were comparatively analyzed. Results: HGT was observed in $41.4 \%$ of RMGs. Original tumor size was larger in the HGT group and recurrence time interval was shorter. Following recurrence, $57.1 \%$ in the HGT group experienced further disease progression, compared to $22.2 \%$ in the nHGT group. Conclusion: A considerable HGT rate in RMGs developed after MG1 was observed. Although HGT was not distinguished from $n H G T$ by radiological features, HGT in $R M G$ was associated with larger initial tumor size and shorter recurrence time interval.
\end{abstract}

Meningioma (MG) is the most prevalent intracranial tumor, representing up to approximately $30 \%$ of all brain tumors (1). The main treatment approach for MG is surgical resection and occasionally, external-beam radiation therapy, if the tumour has a complex anatomical location. Surgical

Correspondence to: Kiyong Na, Department of Pathology, Kyung Hee University Hospital, Kyung Hee University College of Medicine, 26 Kyungheedae-ro, Dongdaemun-gu, Seoul 02447, Republic of Korea. Tel: +82 29588740, Fax: +82 29588744, e-mail: raripapa@gmail.com

Key Words: Meningioma, recurrence, high grade transformation. resection provides a high cure rate in a vast majority of patients with MG, but disease recurrence still remains the major drawback in patients with a complicated clinical course. Large population-based studies have reported that the overall recurrence rate of $\mathrm{MG}$ within 10 years after surgery ranges from $10 \%$ to $32 \%(2,3)$. Patients experiencing recurrent disease show increased incidence of co-morbidity and decreased overall survival (4).

One of the most important factors predicting the recurrence of $\mathrm{MG}$ is histological grade $(2,3,5,6)$. According to the World Health Organization classification, MG grade is classified using a 3-tier system based on histopathological features (7). Over $80 \%$ of $\mathrm{MGs}$ are grade I and the recurrence rate for $\mathrm{MG}$ grade I (MG1) ranges between $7 \%$ to $20 \%$. MG of grade II and III (MG2-3) have relatively lower incidences but much higher recurrence rate which ranges from $30 \%-40 \%$ and $50 \%-80 \%$, respectively (8).

As seen in various types of human neoplasms, the grade of MG may progress into a higher one as the disease itself progresses. It is widely accepted that MG2-3 arises from MG1 as a consequence of genetic evolution, including complex chromosomal gene gains or losses (9). An increasing grade in consecutively obtained $\mathrm{MG}$ tissues through the time of initial diagnosis to disease recurrence have been reported (10).

Recognizing high grade transformation (HGT) in recurrent MG (RMG) is important as it provides information that could be helpful for predicting adverse patient outcomes and adopting a more aggressive disease management. Unfortunately, there are currently no alternative methods to histological examination for grading of MG. However, if clinical observations suggestive of HGT in RMG could be identified this would facilitate the selection of high-risk 
patients for further tissue sampling. To the best of our knowledge, there have been few studies demonstrating a series of RMG with HGT (11). The aim of this study was to characterize the clinicoradiological features of RMG with HGT developed after MG treatment.

\section{Patients and Methods}

Patients. Figure 1 summarizes the study design. The pathology database in Kyung Hee University Hospital (Seoul, Republic of Korea) was queried for all cases of MG, diagnosed between January 1998 and January 2013. A total of 291 patients underwent surgery for primary MG (PMG) and 22/291 patients underwent a second surgery for RMG. To find cases of RMG which were not surgically resected, we reviewed the radiology reports of all 291 patients and found 13 additional cases. Overall, 35/291 (12.0\%) patients experiencing RMG were identified. This study (2019-07-075) was approved by the Institutional Review Board of the Kyung Hee University Hospital.

Review of pathological diagnosis. Three experienced pathologists (K $\mathrm{Na}$, SI Do and JY Sung) reviewed all available hematoxylin and eosin slides of 291 PMG and 29 RMG (more than 1 RMG specimens in 7 patients) with routine microscopic examination and re-classified the tumor grade based on the 2016 WHO classification system (7).

Review of medical records. We thoroughly reviewed the electronic medical records of 35 patients with RMG, from the time between the initial diagnosis and the last follow up. Data at the time of the initial diagnosis included patients' age and sex, tumor site, tumor size, extent of the surgery, and co-morbidity. Routine follow up radiologic examinations were performed at 6,12 and 24 months postoperatively, based on gadolinium-enhanced magnetic resonance image (MRI) or contrast-enhanced computed tomography (CT) when MRI was not feasible. Thereafter, CT/MR follow up was performed at 2-3-year intervals. The primary endpoint was disease recurrence diagnosed by radiological detection of a newly developed lesion or a marked enlargement of remnant tumor. The details that were reviewed at the primary endpoint included patients' age, disease interval from surgery, and detailed imaging findings. The secondary endpoint was death or last follow up. At the secondary end point, total follow up period, disease progression after first recurrence and patients' survival were documented.

Image analysis. The full array of follow up images was randomly presented to two independent neuroradiologists (EK Yeon and EJ Kim) who were blinded to pertinent clinical and radiologic information. Radiological parameters reviewed at the time of recurrence included multiplicity, tumor size, recurrence site in relation to prior location, proximity to major sinus, texture, shape, border, peritumoral edema, intratumoral calcification, signal intensity on T1- and T2 weighted images (WI), pattern of enhancement and invasion of surrounding structures. Peritumoral brain edemas were categorized using 3 scales based on the extent and intensity of the signal surrounding the MG on T2- or fluidattenuated inversion recovery-weighted MRI sequences: 1) mild: absence of or small halo around the perimeter of the MG;2) moderate: extensions to white matter without involving more than twice the volume of the tumor; 3 ) severe: almost entire hemispheric edema. Signal intensities of the lesions were assessed on T1- and T2- WI by comparison with those of cortical grey matter. The patterns of enhancement were classified as either heterogenous or homogenous. Invasion was categorized as none, brain, and surrounding structure.

Statistical analysis. A chi-square test or Fisher's exact test was used to determine whether there was an association between the categorical variables. An independent t-test was used to compare the means between two continuous variables. Univariate survival analyses were performed using the Kaplan-Meier method and a logrank test. Statistical analyses were performed using PASW Statistics for Windows (version 18.0; IBM SPSS, Armonk, NY, USA). Statistical significance was set at a $p$-value $<0.05$.

\section{Results}

Review of histological grade. We reviewed the histological grade in 5/291 (1.7\%) of PMG and 1/29 (3.4\%) of RMG, respectively (Figure 1). Major discrepancy with original diagnosis in $4 / 6$ of revised cases was attributed to the different interpretations regarding brain invasion. We downgraded 3 cases of MG2 into MG1 since they exhibited borders invading adjacent brain parenchyma. One case initially diagnosed as MG1, which exhibited diffuse replacement of brain parenchyma, was upgraded into MG2, based on the identification of inter-tumoral narrow brain parenchymal tissue. Upgrading of MG1 to MG2 in 2 cases was based on mitotic count.

The overall recurrence rate based on the revised diagnosis was as follows: $7.3 \%$ (19/259; MG1), 48.1\% (13/27; MG2) and $60.0 \%(3 / 5 ; \mathrm{MG} 3)$. Histological examination of RMG was available in $89.4 \%(17 / 19)$ of total recurrence cases developed in patients with MG1. Of these, $41.1 \%(7 / 17)$ of the tumors recurred as MG2 and $58.8 \%$ (10/17) recurred as MG1, respectively. We defined 7 patients with RMG2 arising in PMG1 as the HGT group and 10 patients with RMG1 arising in PMG1 as the non-HGT (nHGT) group, respectively. Two cases of RMG in the HGT group showed an abrupt transition between low- and high-grade histological features. Histological examination of RMG was available in $31.3 \%$ of total recurrence cases developed in patients with MG2-3. Of these, $25.0 \%$ (1/4) of MG2 recurred as MG3.

Clinical features of HGT group. Clinical features of the HGT group are summarized in Table I (No. 1-7). The median age at the time of initial diagnosis was 60 years. Male:female ratio was $4: 3$. The majority $(85.7 \%)$ of patients presented with a single mass, with the following anatomical distribution: parasagittal $(n=3)$, convexity $(n=2)$, skull base $(n=1)$ and intraventricular $(n=1)$. One patient presenting with multiple masses had a history of bilateral cerebellopontine angle schwannoma and neurofibromatosis type 2 (No. 6). The median size of the tumors was $5.3 \mathrm{~cm}$. All patients underwent a total or near total resection of the tumor. 


\begin{tabular}{|c|c|c|c|c|c|}
\hline \multicolumn{2}{|c|}{$\begin{array}{l}\text { Pathology database } \\
\text { "Meningioma" }\end{array}$} & \multirow[t]{2}{*}{$\begin{array}{l}\text { Review of image findings } \\
\text { "Recurrent meningioma" }\end{array}$} & \multicolumn{3}{|c|}{ Distribution of tumor grade in recurrent meningioma } \\
\hline & & & Recurrence in Grade I & $19 / 259$ & $(7.3 \%)$ \\
\hline Primary & $n=291$ & Newly developed lesion & Grade I & $10 / 19$ & $(52.6 \%)$ \\
\hline Grade I & $n=258$ & Marked increase in size & Grade II & $7 / 19$ & $(36.8 \%)$ \\
\hline Grade II & $n=28$ & Additional 13 cases & Tissue not submitted & $2 / 19$ & $(10.5 \%)$ \\
\hline \multirow[t]{2}{*}{ Grade III } & $\mathrm{n}=5$ & & Recurrence in Grade II & $13 / 27$ & $(48.1 \%)$ \\
\hline & & Review of histopathology & Grade II & $3 / 13$ & $(23.0 \%)$ \\
\hline Recurrent & $\mathrm{n}=22$ & Primary meningioma & Grade III & $1 / 13$ & $(7.6 \%)$ \\
\hline Grade I & $n=11$ & Grade II $\rightarrow$ Grade I $n=3$ & Tissue not submitted & $9 / 13$ & $(69.2 \%)$ \\
\hline Grade II & $n=9$ & Grade I $\rightarrow$ Grade II $n=2$ & Recurrence in Grade III & $3 / 5$ & $(60.0 \%)$ \\
\hline \multirow[t]{2}{*}{ Grade III } & $n=2$ & Recurrent meningioma & Grade III & $1 / 3$ & $(33.3 \%)$ \\
\hline & & Grade I -> Grade II & Tissue not submitted & $2 / 3$ & $(66.6 \%)$ \\
\hline
\end{tabular}

Figure 1. Study design.
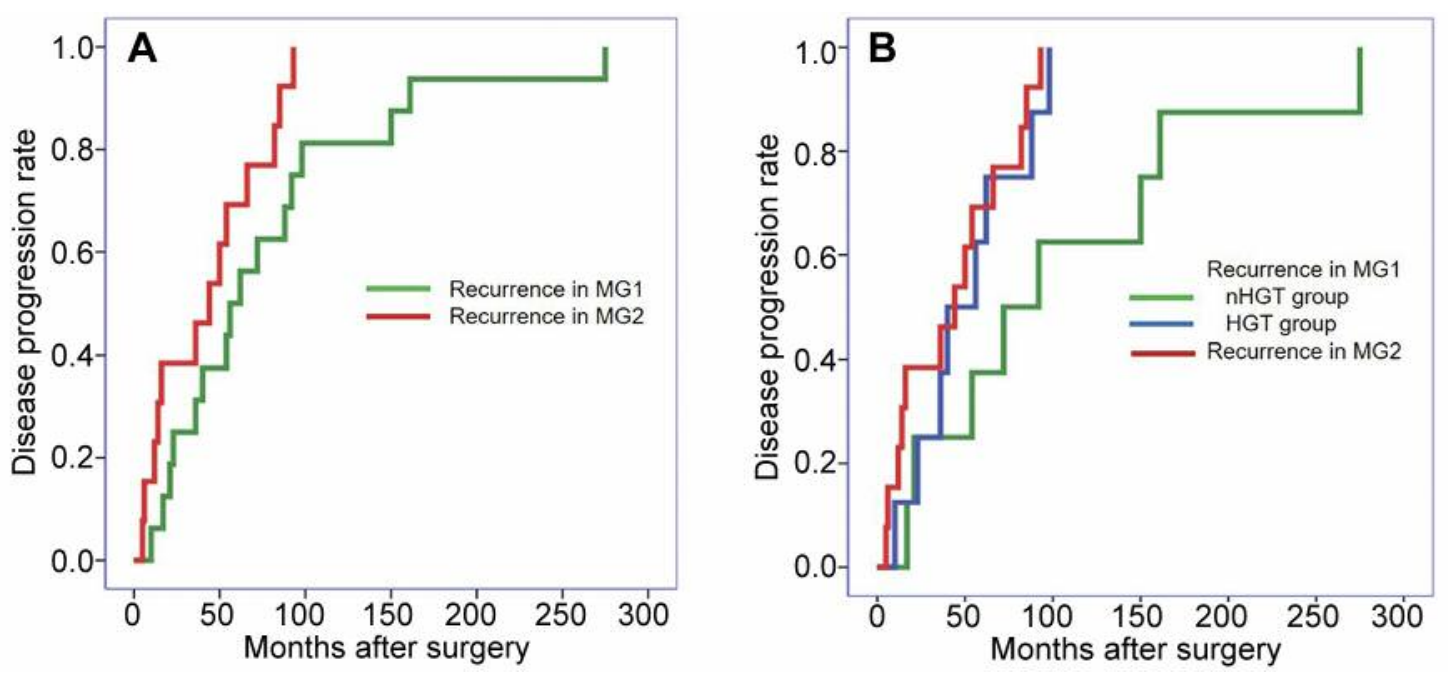

Figure 2. Kaplan-Meier analysis of recurrence-free survival after initial diagnosis. A) Recurrence time interval in the total recurrent cases developed after diagnosis of MG1 and MG2 at their primary tumor. B) Recurrence time interval of patients who were initially diagnosed as MG1 and were further divided into HGT and nHGT groups.

The median interval between the initial treatment and the detection of recurrence was 40 months. The median age at the time of recurrence was 67 years. The median size of the recurrent tumor was $4.0 \mathrm{~cm}$. Two patients exhibited a recurrent tumor in a different location from the primary tumor (No. 4 and 5). One patient with neurofibromatosis type 2 exhibited multiple masses at the time of recurrence.

Clinical features of $n H G T$ group. Clinical features of the nHGT group are summarized in Table I (No. 8-17). The median age at the time of initial diagnosis was 50 years. Male:female ratio was 5:5. The majority $(90.0 \%)$ of patients presented with a single mass with the following anatomical distribution: parasagittal $(n=4)$, skull base $(n=3)$, posterior fossa $(n=2)$ and convexity $(n=1)$. One patient presenting with multiple masses had history of unilateral cerebellopontine angle schwannoma and neurofibromatosis type 2 (No. 13). The median size of the tumors was $3.5 \mathrm{~cm}$. All the patients underwent a total or near total resection of the tumor.

The median interval between the initial treatment and the detection of recurrence was 121 months. The median age at the time of recurrence was 60 years. The median size of the recurrent tumors was $5.1 \mathrm{~cm}$. None of the tumors recurred in a different site from that of the primary tumor. Two patients, including a patient with neurofibromatosis type 2, exhibited multiple masses at the time of recurrence (No. 13 and 14). 
Table I. Clinical features of 17 patients experiencing recurrent meningioma after diagnosis of grade I meningioma.

\begin{tabular}{|c|c|c|c|c|c|c|c|c|c|c|c|}
\hline & \multirow[t]{2}{*}{ Age } & \multirow[t]{2}{*}{ Gender } & \multicolumn{4}{|c|}{ Primary meningioma } & \multirow[t]{2}{*}{ RFS (mo) } & \multicolumn{3}{|c|}{ Recurrent meningioma } & \multirow[t]{2}{*}{ Co-morbidity } \\
\hline & & & Site & Size $(\mathrm{cm})$ & Surgery & Diagnosis & & Size $(\mathrm{cm})$ & Site & Diagnosis & \\
\hline 1 & 50 & M & P, SSS & 5.3 & NTR & MG1 & 10 & 7.4 & P, SSS & MG2 & - \\
\hline 2 & 79 & $\mathrm{~F}$ & C, occipital & 7 & NTR & MG1 & 62 & 3.5 & C, occipital & MG2 & - \\
\hline 3 & 62 & M & P, SSS & 5.4 & NTR & MG1 & 98 & 2.6 & P, SSS & MG2 & - \\
\hline 4 & 43 & M & P, SSS & 6.3 & $\mathrm{TR}$ & MG1 & 23 & 6.9 & $\mathrm{C}$, frontal & MG2 & - \\
\hline 5 & 60 & M & $\mathrm{C}$, parietal & 4.1 & TR & MG1 & 88 & 4.0 & $\mathrm{C}$, frontal & MG2 & - \\
\hline 6 & 48 & $\mathrm{~F}$ & I, trigone & 3.9 & TR & MG1 & 36 & 2.6 & I, trigone & MG2 & NF type 2 \\
\hline 7 & 65 & $\mathrm{~F}$ & S, sphenoid & 5 & $\mathrm{TR}$ & MG1 & 40 & 4.2 & $\mathrm{~S}$, sphenoid & MG2 & - \\
\hline 8 & 60 & M & $\mathrm{PO}, \mathrm{CPA}$ & 3.1 & NTR & MG1 & 56 & 5.2 & $\mathrm{PO}, \mathrm{CPA}$ & MG1 & - \\
\hline 9 & 50 & $\mathrm{~F}$ & P, SSS & 3.9 & TR & MG1 & 150 & 4.7 & $\mathrm{P}, \mathrm{SSS}$ & MG1 & - \\
\hline 10 & 36 & M & $\mathrm{PO}$, tentorium & 1.5 & TR & MG1 & 275 & 8.0 & $\mathrm{PO}$, tentorium & MG1 & - \\
\hline 11 & 50 & $\mathrm{~F}$ & $\mathrm{P}, \mathrm{SSS}$ & 5 & TR & MG1 & 54 & 5.0 & $\mathrm{P}, \mathrm{SSS}$ & MG1 & - \\
\hline 12 & 52 & $\mathrm{~F}$ & S, olfactory & 5.3 & TR & MG1 & 161 & 6.4 & S, olfactory & MG1 & - \\
\hline 13 & 44 & $\mathrm{~F}$ & $\mathrm{P}, \mathrm{SSS}$ & 2 & NTR & MG1 & 72 & 4.7 & P, SSS & MG1 & NF type 2 \\
\hline 14 & 46 & M & S, sphenoid & 5.7 & $\mathrm{TR}$ & MG1 & 92 & 2.4 & S, sphenoid & MG1 & - \\
\hline 15 & 60 & M & P, SSS & 6.4 & NTR & MG1 & 34 & 5.5 & $\mathrm{P}, \mathrm{SSS}$ & MG1 & - \\
\hline 16 & 40 & $\mathrm{~F}$ & S, olfactory & 4 & $\mathrm{TR}$ & MG1 & 217 & 4.8 & S, olfactory & MG1 & - \\
\hline 17 & 59 & M & $\mathrm{C}$, temporal & 2.5 & TR & MG1 & 170 & 5.6 & $\mathrm{C}$, temporal & MG1 & \\
\hline
\end{tabular}

RFS: Recurrence-free survival; MG1: meningioma grade I; MG2: meningioma grade II; NF: neurofibromatosis; TR: total resection; NTR: near total resection; C: convexity; P: parasagittal; S: skull base; PO: posterior fossa; I: intraventricle; SSS: superior sagittal sinus; CPA: cerebellopontine angle.

Comparative analysis of clinical and radiological features between HGT group and nHGT group. Table II summarizes the comparative analysis of clinical and radiological features between the two groups. The size of the primary tumor was significantly larger in the HGT group $(p=0.04)$. Also, the recurrence time interval was significantly shorter in the HGT group $(p=0.03)$. None of the radiological features analyzed in this study showed a significant difference between the HGT and nHGT groups. Although there was no statistical significance $(p=0.154)$, recurrence in a different location from that of the primary tumor was exclusively found in the HGT group.

Patient outcome. Table III summarizes overall follow up results after diagnosis of PMG. In the extended observation period, $41.2 \%$ of patients progressed to MG2 (mean $=96.3 \pm 74.0$ months). Overall annual HGT rate in RMG was $5.1 \%$ per patient-year. Median follow up period after detection of recurrence in the HGT group was 50 months (range $=12-143$ months). During the follow up period, $57.1 \%$ of the patients in the HGT group experienced further disease progression including lung metastasis $(n=1)$ and local recurrence $(n=3)$. One patient with metastasis to the lungs died from the disease within 1 month. The remaining 6 patients were alive at the last follow up. The median follow-up period after detection of recurrence in the nHGT group was 25 months (range $=6-180$ months). One of the 10 patients died of a surgical complication. From the remaining 9 patients, $22.2 \%$ experienced further local recurrence. All 9 patients were alive at the last follow up.

Comparison of recurrence time interval between MGl and $M G 2$. We compared the recurrence time interval in the total recurrent cases developed after diagnosis of MG1 and MG2 at their primary tumor (Figure 2A). Median time interval to recurrence in patients with MG2 was significantly shorter, compared to patients with MG1 (36 vs. 72 months, respectively; $p=0.009$ ).

Next, the patients who were initially diagnosed as MG1 were further divided into HGT and nHGT groups (Figure 2B). Median recurrence time interval in the nHGT group was significantly longer than that in the HGT group (121 vs. 40 months, respectively; $p=0.011)$ and in patients with MG2 (121 vs. 36 months, respectively; $p=0.007$ ). Median recurrence time interval in the HGT group was not significantly different from that in patients with MG2 (40 vs. 36 months, respectively; $p=0.622$ ).

\section{Discussion}

We observed that 19/259 (7.3\%) patients with MG1 experienced disease recurrence during a mean follow up period of 96.3 months. The cumulative recurrence rate of MG1 was estimated at $0.91 \%$ per patient-year. These results are in line with the results of a previous systematic review and meta-analysis of recurrence rate extracted from 1539 
Table II. Comparison of clinical features between HGT group and nHGT group.

\begin{tabular}{|c|c|c|c|}
\hline & HGT $(n=7)$ & nHGT $(\mathrm{n}=10)$ & $p$-Value \\
\hline \multicolumn{4}{|l|}{ Primary meningioma } \\
\hline Age at diagnosis (years, mean \pm SD) & $58.1 \pm 12.2$ & $49.7 \pm 8.3$ & 0.113 \\
\hline Gender (M:F) & $4: 3$ & $5: 5$ & 1.000 \\
\hline Tumor size $(\mathrm{cm}$, mean $\pm \mathrm{SD})$ & $5.2 \pm 1.1$ & $3.8 \pm 1.6$ & 0.041 \\
\hline \multicolumn{4}{|l|}{ Multiplicity } \\
\hline Single & 6 & 9 & 1.000 \\
\hline Multiple & 1 & 1 & \\
\hline \multicolumn{4}{|l|}{ Extent of the surgery } \\
\hline Total resection & 4 & 7 & 0.643 \\
\hline Near total resection & 3 & 3 & \\
\hline \multicolumn{4}{|l|}{ Recurrent meningioma } \\
\hline Age at recurrence & $62.2 \pm 13.7$ & $60.4 \pm 6.7$ & 0.712 \\
\hline Disease interval (months; mean \pm SD) & $51 \pm 32.9$ & $128.1 \pm 79.4$ & 0.031 \\
\hline Tumor size $(\mathrm{cm}$, mean $\pm \mathrm{SD})$ & $4.5 \pm 1.9$ & $5.2 \pm 1.4$ & 0.393 \\
\hline \multicolumn{4}{|l|}{ Recurred site } \\
\hline Same with primary & 5 & 10 & 0.154 \\
\hline Different from primary & 2 & 0 & \\
\hline \multicolumn{4}{|l|}{ Multiplicity } \\
\hline Single & 6 & 8 & 1.000 \\
\hline Multiple & 1 & 2 & \\
\hline \multicolumn{4}{|l|}{ Follow-up after recurrence } \\
\hline Available & 7 & 9 & \\
\hline Not available & 0 & 1 (Post op death) & \\
\hline Follow up period (months; mean \pm SD) & $62.4 \pm 54.2$ & $45.2 \pm 57.2$ & \\
\hline Disease progression rate & $57 \%$ & $22 \%$ & \\
\hline
\end{tabular}

Table III. Patient outcomes.

\begin{tabular}{|c|c|c|c|c|c|c|c|}
\hline No. & Age & Diagnosis (Initial) & RFS (mo) & Diagnosis (Recurrent) & $\mathrm{FU}(\mathrm{mo})$ & Status & Recurrence site \\
\hline 1 & 50 & MG1 & 10 & MG2 & 50 & Died of progressive disease & Lung Mets \\
\hline 2 & 79 & MG1 & 62 & MG2 & 12 & Alive with stable disease & \\
\hline 3 & 62 & MG1 & 98 & MG2 & 143 & Alive with progressive disease & Local \\
\hline 4 & 43 & MG1 & 23 & MG2 & 107 & Alive with progressive disease & Local \\
\hline 5 & 60 & MG1 & 88 & MG2 & 7 & Alive with stable disease & \\
\hline 6 & 48 & MG1 & 36 & MG2 & 18 & Alive with stable disease & \\
\hline 7 & 65 & MG1 & 40 & MG2 & 100 & Alive with progressive disease & Local \\
\hline 8 & 60 & MG1 & 56 & MG1 & None & Post operation death & \\
\hline 9 & 50 & MG1 & 150 & MG1 & 52 & Alive with progressive disease & Local \\
\hline 10 & 36 & MG1 & 275 & MG1 & 25 & Alive with stable disease & \\
\hline 11 & 50 & MG1 & 54 & MG1 & 194 & Alive with progressive disease & Local \\
\hline 12 & 52 & MG1 & 161 & MG1 & 27 & Alive with stable disease & \\
\hline 13 & 44 & MG1 & 72 & MG1 & 19 & Alive with stable disease & \\
\hline 14 & 46 & MG1 & 92 & MG1 & 6 & Alive with stable disease & \\
\hline 15 & 60 & MG1 & 34 & MG1 & 32 & Alive with stable disease & \\
\hline 16 & 40 & MG1 & 217 & MG1 & 24 & Alive with stable disease & \\
\hline 17 & 59 & MG1 & 170 & MG1 & 25 & Alive with stable disease & \\
\hline
\end{tabular}

RFS: Recurrence-free survival; MG1: meningioma grade I; MG2: meningioma grade II; FU: follow up.

patients with MG1, where cumulative recurrence rate ranged from 0.00 to $2.36 \%$ per patient-year (6). Although the recurrence rate of MG1 is low, there was a considerable proportion of HGT in the recurrent tumors. In our series,
41.1\% (7/17) of RMG developed in patients with MG1 were accompanied by HGT, which was slightly higher than the 14 to $29 \%$ HGT rate in RMG reported in earlier studies (12-14). 
In an effort to investigate the diagnostic signs of HGT in RMG, we comparatively analyzed various clinicoradiological factors between the HGT and nHGT groups and found that the recurrence time interval was shorter in the HGT group compared to the nHGT group. Also, by comparing recurrence time interval of patients with MG2, we found that the HGT group had a similar recurrence time interval compared to patients with MG2, suggesting that HGT should be suspected in patients with rapid growing tumors after initial treatment. In line with our findings, Champeaux et al. (15) demonstrated that HGT in RMG is associated with surgical recurrence-free survival. Furthermore, Zhao et al. (16) reported that RMG with HGT had a shorter progressionfree survival than MG2-3 at their initial diagnosis.

Additionally, tumor size at initial diagnosis was larger in the HGT group. Results from previous studies support our observations, as the size of MG has been consistently reported as a predisposing risk factor for recurrence (17-20). It is thought that the difficulty in achieving a complete resection as well as the high proliferative capacity of the tumour cells help explain these observations. Based on the recent genetic studies suggesting that $\mathrm{MG}$ progression is the result of a multistep genetic mutation (10), it is reasonable to consider that there is an increased chance of genetic changes leading to HGT in tumors with high cellularity.

We also analyzed the radiological features of tumors and peri-tumoral changes, but none of the parameters showed significant differences between the HGR and nHGT groups. One interesting finding was that the recurrent tumor occurred in a different location from that of the primary tumor $(2 / 7)$ exclusively in the HGT group (28\% vs. $0 \% ; p=0.154)$, although without achieving statistical significance.

With further follow up, we confirmed that the clinical outcome of the HGT group is unfavorable compared to that of the nHGT group. Long term follow-up showed that $57.1 \%$ of patients in the HGT group continuously experienced disease progression, including lung metastasis and local recurrence, in contrast with the nHGT group which only showed further disease progression in $22.2 \%$ of the cases. These results emphasize the importance of diagnosis of HGT in RMG for predicting patient outcome as well as determining clinical management. Considering that we found no reliable radiological features useful for the diagnosis of HGT in RMG, a careful evaluation of clinical history is essential and active tissue sampling should be strongly considered for patients with an aggressive clinical course.

There were certain limitations to this study. First, a selection bias was inevitable and inherent due to its retrospective and single center study design. Patients with RMG treated with radiation therapy and those who did not agree with repeated surgery were not included due to the lack of pathologic tissue confirmation. Second, follow-up protocols such as imaging modality or exam interval, were not uniformly applied within the study cohort. Finally, the small number of study subjects having a relatively low probability of RMG after MG1, may have skewed our discovery of clinical factors for HGT prediction. Results from our study need further validation in a large-scale study.

In summary, although recurrence is not common in MG1, RMG developed after MG1 has a considerably high rate of HGT. Without reliable radiological signs of HGT, active tissue confirmation is mandatory, especially in patients with RMG accompanied by aggressive clinical features such as large primary tumor or short disease recurrence interval.

\section{Conflicts of Interest}

The Authors have no conflicts of interest to declare regarding this study.

\section{Authors' Contributions}

All Authors made substantial contributions to the conception and design of the study, data acquisition, analysis and interpretation, as well as drafting the manuscript, revising the article critically for intellectual content, and providing final approval of the version to be published.

\section{Acknowledgements}

This research was supported by the Basic Science Research Program through the National Research Foundation of Korea (NRF) funded by the Ministry of Science, ICT and Future Planning (2017R1A2B4007704).

\section{References}

1 Ostrom QT, Gittleman H, Fulop J, Liu M, Blanda R, Kromer C, Wolinsky Y, Kruchko C and Barnholtz-Sloan JS: CBTRUS statistical report: primary brain and central nervous system tumors diagnosed in the United States in 2008-2012. Neuro Oncol 17: iv1iv62, 2015. PMID: 26511214. DOI: 10.1093/neuonc/nov189

2 Adegbite AB, Khan MI, Paine KW and Tan LK: The recurrence of intracranial meningiomas after surgical treatment. J Neurosurg 58: 51-56, 1983. PMID: 6847909. DOI: 10.3171/jns.1983.58.1.0051

3 Mirimanoff RO, Dosoretz DE, Linggood RM, Ojemann RG and Martuza RL: Meningioma: analysis of recurrence and progression following neurosurgical resection. J Neurosurg 62: 18-24, 1985. PMID: 3964853. DOI: 10.3171/jns.1985.62.1.0018

4 Yang SY, Park CK, Park SH, Kim DG, Chung YS and Jung HW: Atypical and anaplastic meningiomas: prognostic implications of clinicopathological features. J Neurol Neurosurg Psychiatry 79: 574-580, 2008. PMID: 17766430. DOI: 10.1136/jnnp.2007.121582

5 Gallagher MJ, Jenkinson MD, Brodbelt AR, Mills SJ and Chavredakis E: WHO grade 1 meningioma recurrence: Are location and Simpson grade still relevant? Clin Neurol Neurosurg 141: 117121, 2016. PMID: 26780494. DOI: 10.1016/j.clineuro.2016.01.006

6 Lam Shin Cheung V, Kim A, Sahgal A and Das S: Meningioma recurrence rates following treatment: a systematic analysis. J Neurooncol 136: 351-361, 2018. PMID: 29143273. DOI: 10.1007/s11060-017-2659-6 
7 Louis DN, Perry A, Reifenberger G, von Deimling A, FigarellaBranger D, Cavenee WK, Ohgaki H, Wiestler OD, Kleihues P and Ellison DW: The 2016 World Health Organization classification of tumors of the central nervous system: a summary. Acta Neuropathol 131: 803-820, 2016. PMID: 27157931. DOI: 10.1007/s00401-016-1545-1

8 Saraf S, McCarthy BJ and Villano JL: Update on meningiomas. Oncologist 16: 1604-1613, 2011. PMID: 22028341. DOI: 10.1634/theoncologist.2011-0193

9 Choy W, Kim W, Nagasawa D, Stramotas S, Yew A, Gopen Q, Parsa AT and Yang I: The molecular genetics and tumor pathogenesis of meningiomas and the future directions of meningioma treatments. Neurosurg Focus 30: E6, 2011. PMID: 21529177. DOI: $10.3171 / 2011.2$.FOCUS1116

10 Al-Mefty O, Kadri PA, Pravdenkova S, Sawyer JR, Stangeby C and Husain $\mathrm{M}$ : Malignant progression in meningioma: documentation of a series and analysis of cytogenetic findings. J Neurosurg 101: 210-218, 2004. PMID: 15309910. DOI: 10.3171/jns.2004.101.2.0210

11 Champeaux C, Wilson E, Shieff C, Khan AA and Thorne L: WHO grade II meningioma: a retrospective study for outcome and prognostic factor assessment. J Neurooncol 129: 337-345, 2016. PMID: 27311726. DOI: 10.1007/s11060-016-2181-2

12 Jellinger K and Slowik F: Histological subtypes and prognostic problems in meningiomas. J Neurol 208: 279-298, 1985. PMID: 50413.

13 Rohringer M, Sutherland GR, Louw DF and Sima AA: Incidence and clinicopathological features of meningioma. J Neurosurg 71: 665-672, 1989. PMID: 2809720. DOI: 10.3171/jns.1989.71.5.0665

14 Violaris K, Katsarides V, Karakyriou M and Sakellariou P: Surgical outcome of treating grades II and III meningiomas: a report of 32 cases. Neurosci J 2013: 706481, 2013. PMID: 26317097. DOI: $10.1155 / 2013 / 706481$

15 Ohta $M$ and Takeshita I: A case of recurrent convexity meningioma with malignant transformation 26 years after total tumor removal. No Shinkei Geka 29: 81-85, 2001. PMID: 11218772 .

16 Jääskeläinen J, Haltia M, Laasonen E, Wahlström T and Valtonen $\mathrm{S}$ : The growth rate of intracranial meningiomas and its relation to histology. An analysis of 43 patients. Surg Neurol 24: 165-172, 1985. PMID: 4012573. DOI: 10.1016/0090-3019(85)90180-6

17 Jääskeläinen J, Haltia M and Servo A: Atypical and anaplastic meningiomas: radiology, surgery, radiotherapy, and outcome. Surg Neurol 25: 233-242, 1986. PMID: 3945904. DOI: $10.1016 / 0090-3019(86) 90233-8$
18 Simpson D: The recurrence of intracranial meningiomas after surgical treatment. J Neurol Neurosurg Psychiatry 20: 22-39, 1957. PMID: 13406590. DOI: 10.1136/jnnp.20.1.22

19 Anthofer J, Seidel-Schulz R, Proescholdt M, Brawanski A and Schebesch KM: Meningiomas adjacent to major venous sinuses clinical outcome and recurrence. World Neurosurg 104: 560-566, 2017. PMID: 28512040. DOI: 10.1016/j.wneu.2017.05.025

20 Hortobágyi T, Bencze J, Varkoly G, Kouhsari MC and Klekner Á: Meningioma recurrence. Open Med (Wars) 11: 168-173, 2016. PMID: 28352788. DOI: 10.1515/med-2016-0032

21 Park S, Cha YJ, Suh SH, Lee IJ, Lee KS, Hong CK and Kim JW: Risk group-adapted adjuvant radiotherapy for WHO grade I and II skull base meningioma. J Cancer Res Clin Oncol 145: 1351-1360, 2019. PMID: 30953136. DOI: 10.1007/s00432-01902891-6

22 Tao X, Wang K, Dong J, Hou Z, Wu Z, Zhang J and Liu B: Clinical features, surgical management, and prognostic factors of secretory meningiomas: a single-center case series of 149 patients. J Neurooncol 136: 515-522, 2018. PMID: 29143274. DOI: $10.1007 / \mathrm{s} 11060-017-2671-\mathrm{x}$

23 Christensen D, Laursen $\mathrm{H}$ and Klinken L: Prediction of recurrence in meningiomas after surgical treatment. A quantitative approach. Acta Neuropathol 61: 130-134, 1983. PMID: 6637397. DOI: 10.1007/bf00697392

24 Champeaux C, Houston D and Dunn L: Atypical meningioma. A study on recurrence and disease-specific survival. Neurochirurgie 63: 273-281, 2017. PMID: 28882609. DOI: 10.1016/j.neuchi.2017.03.004

25 Zhao P, Hu M, Zhao M, Ren X and Jiang Z: Prognostic factors for patients with atypical or malignant meningiomas treated at a single center. Neurosurg Rev 38: 101-107, 2015. PMID: 25139398. DOI: $10.1007 / \mathrm{s} 10143-014-0558-2$
Received September 24, 2019

Revised October 8, 2019

Accepted October 10, 2019 